\title{
Peak power \& average power scaling via fourier domain OPA (FOPA)
}

\author{
Bruno E. Schmidt ${ }^{1}$, Vincent Gruson $^{2,3}$, Philippe Lassonde ${ }^{2}$, Guilmot Ernotte $^{2}$, Heide \\ Ibrahim $^{2}$, Denis Ferachou ${ }^{1,2}$, Arvid Hage ${ }^{4}$, Torsten $\mathrm{Mans}^{4}$, Lou Di Mauro ${ }^{3}$, Paul B. \\ Corkum $^{5}$, Hans Jakob Wörner ${ }^{6}$, and François Légaré ${ }^{2}$ \\ ${ }^{1}$ few-cycle Inc., 2890 Rue de Beaurivage, Montreal, H1L 5W5, Qc, Canada \\ ${ }^{2}$ INRS-EMT, 1650 Blvd. Lionel Boulet, Varennes, J3X 1S2, Qc, Canada \\ ${ }^{3}$ Department of Physics, The Ohio State University, Columbus, Ohio 43210, USA. \\ ${ }^{4}$ Amphos GmbH, Kaiserstraße 100, 52134 Herzogenrath, Germany \\ ${ }^{5}$ Joint Attosecond Science Laboratory, University of Ottawa Ottawa K1N 5A2, Canada \\ ${ }^{6} \mathrm{ETH}$ - Zürich, Vladimir-Prelog-Weg 2, 8093 Zürich, Switzerland
}

\begin{abstract}
FoTaking advantage of pulse shortening upon amplification, we demonstrate $2.5 \mathrm{TW}$ pulses $(30 \mathrm{~mJ}, 2$ cycle, $1.8 \mu \mathrm{m}$ ) based on TiSa pumping, while for boosting average power we utilize a $500 \mathrm{~W}$ Yb platform for pump and seed pulses.
\end{abstract}

\section{Introduction}

One important application of Fourier domain nonlinear optics [1] is the optical parametric amplification of few-cycle pulses with FOPA [2]. It enables simultaneous upscaling of pulse energy and bandwidth while this denotes a trade-off for its time domain counterparts like OPCPA. Another grand challenge in contemporary laser science is average power scaling. We will discuss our progress on both FOPA design for high energy operation and the average power boosting based on an $\mathrm{Yb}$ Innoslab pump laser. We will present experimental results for high peak power IR pulses in the 2-cycle regime at $1.8 \mu \mathrm{m}$ wavelength carrying $30 \mathrm{~mJ}$ of pulse energy $(>2 \mathrm{TW})$. Furthermore, we present a $500 \mathrm{~W}$ average power $\mathrm{Yb}$ pump laser with $50 \mathrm{~mJ}$ pulse energy at $10 \mathrm{kHz}$ repetition rate and $1.5 \mathrm{ps}$ pulse duration. The system features unparalleled pointing and power stability in the sub-\% range.

\section{Experimental results}

The peak power scaling of few-cycle pulses is facilitated by the separation ansatz of FOPA which allows one to break down a big task into smaller sub-problems. Due to the frequencies's spatial separation in the Fourier plane (FP) of a $4 \mathrm{f}$ setup, the amplifier output properties are not restricted by the performance of a single crystal but by the number of different individually optimized crystals. Figure 1 summarizes results obtained from a twocrystal FOPA pumped by a ps TiSa beam carrying $250 \mathrm{~mJ}$ of energy [3]. Figs 1a) and 1b) 
show that the good input beam quality after the hollow-core fiber compressor is maintained until maximum amplification. Looking at the retrieved SHG-FROG intensity for seed (Fig. 1c) and amplified pulses (Fig. 1d), however, reveals a surprise. The input pulse duration has shortened from 2.5 cycles to 1.9 cycles upon amplification in the FOPA. The reason can be seen when comparing the seed (gray curve In Fig. 1e) and the amplified spectra (colored curves in Fig. 1e). While the wings in the seed spectrum are close to the noise level of the poor IR spectrometer, they get amplified stronger compared to the center of the spectrum. The spectral dip in thee center arises from the joint of the two crystals in the FP. Thus, the experimental gain function (Fig. 1f) of the two cycle spectrum raises in the spectral wings. This tailoring of the spectral gain function is acieved by controling the spatial pump beam profile of the TiSa pump laser in the FP [3].

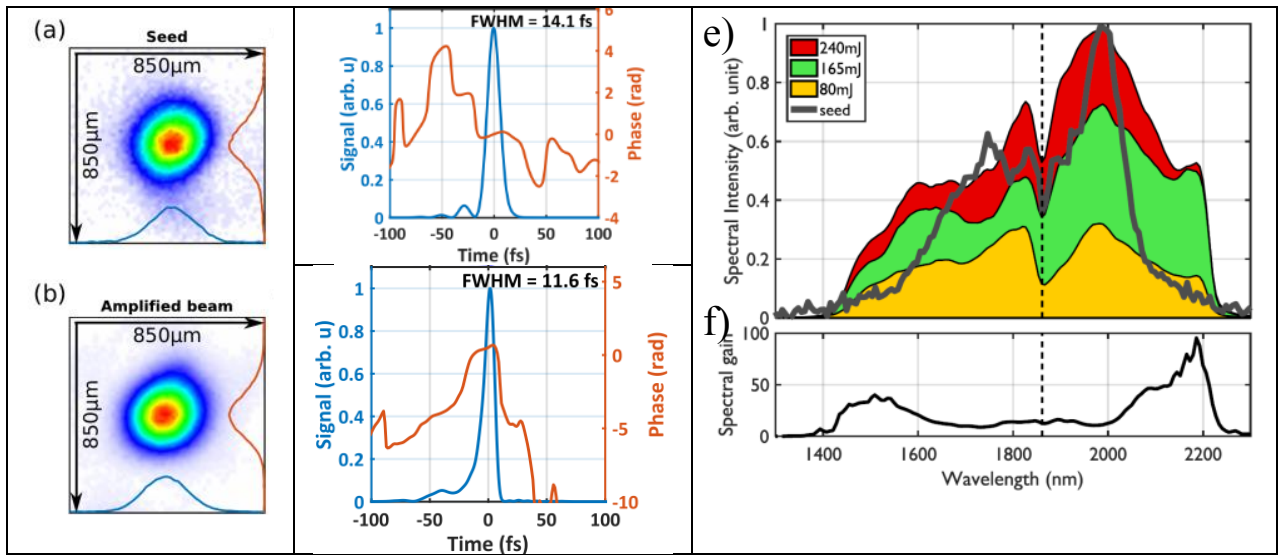

Fig. 1: Input and output specifications for the 1.9cycle, 2.5TW FOPA. Figs a) \& b) display focal spot images,

c) \& d) temporal intensity profiles and e) shows the spectral gain evolution up to $240 \mathrm{~mJ}$ of TiSa pump energy.

To achieve average power scaling, we moved from the TiSa pumping to an $\mathrm{Yb}$ Innoslab laser (Amphos $\mathrm{GmbH}$ ) because of the superior thermal properties of the crystal host material. The main advantages of this type of pump laser are the high gain obtained with a compact multipass design and the excellent properties even at 500W output (see Fig. 2) [4]. Remarkably, an energy stability of about $0.25 \%$ RMS fluctuations is measured over a period of $8 \mathrm{~h}$. Furthermore, the beam is of very good spatial quality. The $\mathrm{M}^{2}$ of the compressed $500 \mathrm{~W}$ output is 1.1 in both directions. A high stability is essential since we split off a small fraction of the $\mathrm{Yb}$ beam at an early stage prior to the main InnoSlab amplifier and booster. A total of only $16 \mathrm{~m}$ beam path through amplifier \& compressor will enable to use the same oscillator pulse for self-seeding and final pumping. To get broadband IR seed pulses, we first generate white light in a YAG plate and simultaneously compress \& selected spectral components with a $4 \mathrm{f}$ setup. The $4 \mathrm{f}$ setup also allows to control the timing and polarization between different white light components prior to their difference frequency generation (DFG) [5].

Since we aim to derive 2-cycle IR pulses directly from the ps pump laser, we compressed the VIS pulses (10fs@700nm, 14fs@600nm) to a duration that supports 2 cycle IR pulses. Consequently, when we perform DFG of the $14 \mathrm{fs}, 620 \mathrm{~nm}$ pulses with a narrowband part at $900 \mathrm{~nm}$ we achieve an IR spectrum centered at $2 \mu \mathrm{m}$ wavelength that spans from $1.4-2.4 \mu \mathrm{m}$, see Fig. 2d).

This spectrum will be further amplifeid in a chain of FOPA stage, ultimately pumped by $500 \mathrm{~W}$ of avergae power 


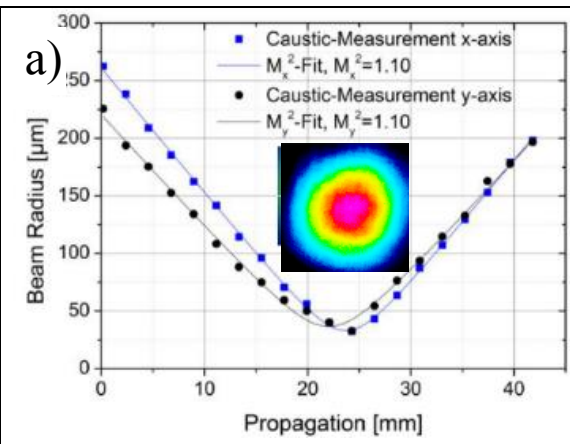

Spatial beam quality at $500 \mathrm{~W}$ compressed output

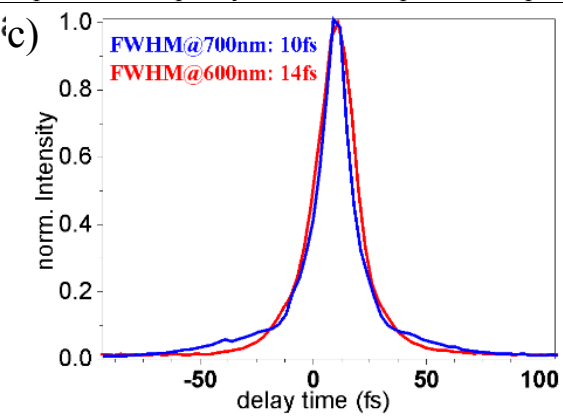

ps driven WL generation \& compression

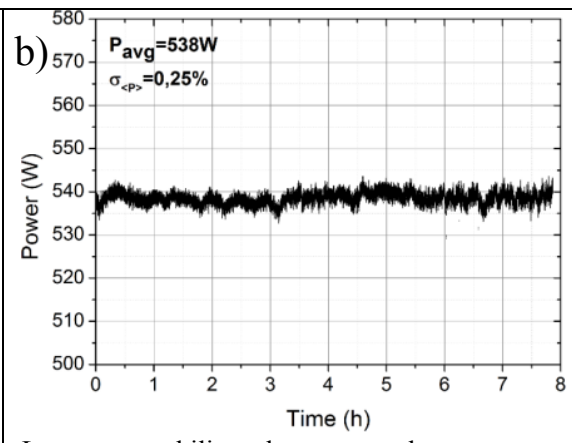

Long term stability subsequent to the compressor

d)

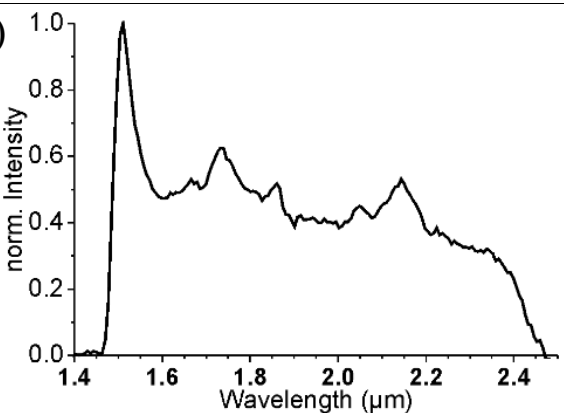

Fig. 2: Characterization of the $500 \mathrm{~W}$ output (a \&b) and of the white light driven with $3 \mu \mathrm{J}$, ps pulses in c). IR seed pulses are derived through DFG, generated by selecting different spectral components of the white light (d).

\section{Conclusion}

We explain and demonstrate how frequency domain nonlinear optics enables the linear transfer of arbitrary phase functions as well as the decoupling of amplitudes and phases and why this is not possible in time domain interaction. A powerful application is deep UV shaping at $207 \mathrm{~nm}$.

\section{References}

[1] Schmidt et al., "Decoupling Frequencies, Amplitudes and Phases in Nonlinear Optics“, Scientific reports 7, 7861 (2017).

[2] Schmidt et al., "Frequency domain optical parametric amplification," Nature Com., 5, 3643 (2014).

[3] Froehly et al., "Progress in Optics," 20, 63-153 (Elsevier, 1983).

[4] Schmidt et al., "Highly stable, $54 \mathrm{~mJ}$ Yb-InnoSlab laser platform at $0.5 \mathrm{~kW}$ average power," Opt. Exp. 25, 17549 (2017).

[5] Ernotte et al., "Frequency domain tailoring for intra-pulse frequency mixing," Opt. Exp. 24, 24225 (2016). 